\title{
Constraining Theories of Embodied Cognition
}

\author{
Arthur B. Markman ${ }^{1}$ and C. Miguel Brendl ${ }^{2}$ \\ ${ }^{1}$ University of Texas and ${ }^{2}$ INSEAD, Fontainebleau, France
}

\begin{abstract}
Influences of perceptual and motor activity on evaluation have led to theories of embodied cognition suggesting that putatively complex judgments can be carried out using only perceptual and motor representations. We present an experiment that revisited a movement-compatibility effect in which people are faster to respond to positive words by pulling a lever than by pushing a lever and are faster to respond to negative words by pushing than by pulling. We demonstrate that the compatibility effect depends on people's representation of their selves in space rather than on their physical location. These data suggest that accounting for embodied phenomena requires understanding the complex interplay between perceptual and motor representations and people's representations of their selves in space.
\end{abstract}

In both cognitive and social psychology, there has been significant interest in the relationship between thought on the one hand and perception and action systems on the other (Barsalou, 1999; Barsalou, Niedenthal, Barbey, \& Ruppert, 2003; Glenberg, 1997; Wilson, 2002). Many studies within this embodied-cognition approach demonstrate that performing actions associated with a particular valence leads to compatibility effects in subsequent judgments. For example, Strack, Martin, and Stepper (1988) had people hold a pen in their mouths (with the tip facing outward) using their lips (which leads to a frowning posture of the lips) or their teeth (which leads to a smiling posture). While doing this, the participants evaluated a series of cartoons. Smileposture participants rated the cartoons as funnier than frownposture participants did.

In the present study, we were interested in the influence of evaluation on speed of motor movements. We examined this relationship in order to explore the tenets of the embodied-cognition approach in detail. Wilson (2002) suggested that the represen-

Address correspondence to Arthur B. Markman, Department of Psychology, University of Texas, 1 University Station, A8000, Austin, TX 78712; e-mail: markman@psy.utexas.edu. tations used by the perception and action systems are necessary for understanding higher-level cognitive processes. Some theorists speculate that perceptual and motor representations are sufficient for understanding cognition, so that cognitive processes can be explained by appealing only to representations that are tied to perceptual and motor modalities (Barsalou et al., 2003; Glenberg, 1997; Prinz, 2002).

\section{ARM MOVEMENTS AND EVALUATIONS}

Many studies have found that movements of the arm are related to people's evaluations. According to this view, pulling movements of the arm are associated with approaching desired objects, and pushing movements of the arm are associated with avoiding undesired objects (Cacioppo, Priester, \& Bernston, 1993; Chen \& Bargh, 1999; Solarz, 1960). For example, Chen and Bargh (1999) showed people words on a computer screen and asked them either to pull or to push a lever to signal the onset of each word. Pulling movements were faster for positive words than for negative words. In contrast, pushing movements were faster for negative words than for positive words. Some theorists have suggested that there are automatic connections between perceptions and motor movements (e.g., Dijksterhuis \& Bargh, 2001) and, indeed, interactions of this type are consistent with such a view.

However, the results of studies using body movements are not completely consistent regarding the relationship between direction of movement and evaluation. For example, Wentura, Rothermund, and Bak (2000) instructed participants to either push a button (an approach response) or withdraw their hand from a button (an avoidance response) as soon as a stimulus was presented. Pressing the button was faster for positive stimuli than for negative ones, but withdrawing the hand from the button was faster for negative stimuli than for positive ones. Given the way participants were seated, pressing the button required a movement away from the body, and withdrawing required a movement toward the body. This reversal of previous findings is inconsistent with an account positing a direct 
connection between body movements and evaluation, but is consistent with an early study by Münsterberg (1892).

Many theories of embodied cognition do not assume direct connections between perception and motor action (Barsalou et al., 2003; Glenberg, 1997; Prinz, 2002), but rather assume that all cognitive representations are tied to perceptual or motor modalities. This richer embodied view is silent on what perceptual or motor representations would cause the observed reversal. Thus, data bearing on this issue would permit elaboration of richer theories of embodied cognition by specifying the ways that cognitive processing integrates conceptual information with perceptual and motor states.

To explore this issue, we began by observing that evaluative movements involve moving objects toward or away from the "self," which is assumed to be located in one's body. Although this assumption is reasonable, it is important to ask whether these two confounded concepts-the representation of the location of the self in space and the representation of one's body in space_can be deconfounded. A natural starting point for embodied theories is that people represent the self as located in the body, and so data suggesting that people can represent the self as being separate from the body would require extensions of these theories.

We constructed a variant of Chen and Bargh's task in which participants' representations of themselves were separated from their representations of their bodies. The setup is illustrated in Figure 1. Each participant sat at a computer screen depicting a corridor receding in depth. The participant's name (representing the self) was presented in the center of the corridor, and words to be evaluated were shown either further away or nearer than the name. In some blocks of trials, participants were instructed to move the joystick in the direction from the word toward their name if the word was positive and away from their name if the word was negative (left side of Fig. 1). In other blocks, participants moved the joystick in the direction from the word away from their name if the word was positive and toward their name if the word was negative (right side of Fig. 1).

When the word was far away in the corridor, participants' representations of self and body were in the same position relative to the stimulus word. In this case, we expected participants to be faster to pull positive words toward their name than to push them away, ${ }^{1}$ and to be faster to push negative words away from their name than to pull them toward it. This finding would replicate Chen and Bargh's (1999) previous findings.

The critical trials were those on which the stimulus word appeared near the participant. In this case, pushing a positive word toward the name involved pushing it toward a nonphysical representation of self and away from the body. Pulling a negative

\footnotetext{
${ }^{1}$ The words on the screen did not actually move. We use this phrasing as a shorthand to refer to moving the joystick in the direction toward or away from the name. In later studies with a slightly different methodology, the word moved toward or away from the name following the response. This manipulation did not influence the strength of the effect; its size was the same as reported here.
}

word away from the name involved pulling it away from a nonphysical representation of self and toward the body. There were two possible outcomes in this condition. If evaluations are connected to movement representations directly, then people would be faster to pull positive words toward their bodies and to push negative words away from their bodies regardless of the position of their names. In contrast, if body movements are made relative to a person's representation of self, then positive words would be moved more quickly toward the name than away from the name and negative words would be moved more quickly away from the name than toward the name.

\section{METHOD}

Participants were 108 German-speaking students at the University of Konstanz, Germany. Two participants with dyslexia, 7 who were not native speakers of German, and 8 who needed but did not have corrective eyewear were excluded. The data from 91 participants were analyzed.

The primary independent variables in this study were valence (positive vs. negative words), movement direction (push vs. pull), and instruction set (positive toward/negative away vs. positive away/negative toward). Valence and movement direction were manipulated within subjects, and instruction set was manipulated between subjects. The dependent variable was response time to initiate the movement of the lever.

Stimuli were 23 positive and 23 negative German words drawn from those used by Fazio, Sanbonmatsu, Powell, and Kardes (1986) and published in Bargh, Chaiken, Govender, and Pratto (1992). Valence of the words was verified for the German sample by thermometer-scale ratings of how cold and warm the words are (Brendl, Markman, \& Messner, in press, Study 1).

Participants sat at a computer and grasped an arm lever with their dominant hand. They were told that their first name would appear in the middle of a corridor depicted on the screen and that another (valenced) word would appear shortly afterward, either in front of their name or behind it. Participants were randomly assigned to either the positive toward/negative away condition (in which they were instructed to move the lever toward their name for positive words and away from their name for negative words; Fig. 1, left side) or the positive away/ negative toward condition (in which they were instructed to move the lever away from their name for positive words and toward their name for negative words; Fig. 1, left side). Before the experimental trials, participants were given the opportunity to practice, first with the words bad and good, and then with various valenced words. Feedback was given during these practice trials. There were 100 experimental trials divided into four blocks separated by pauses. Pauses were followed by 2 warm-up trials.

Response times (in milliseconds) were calculated from the moment of onset of the stimulus to the point when the lever was moved $0.208 \mathrm{~mm}$. 


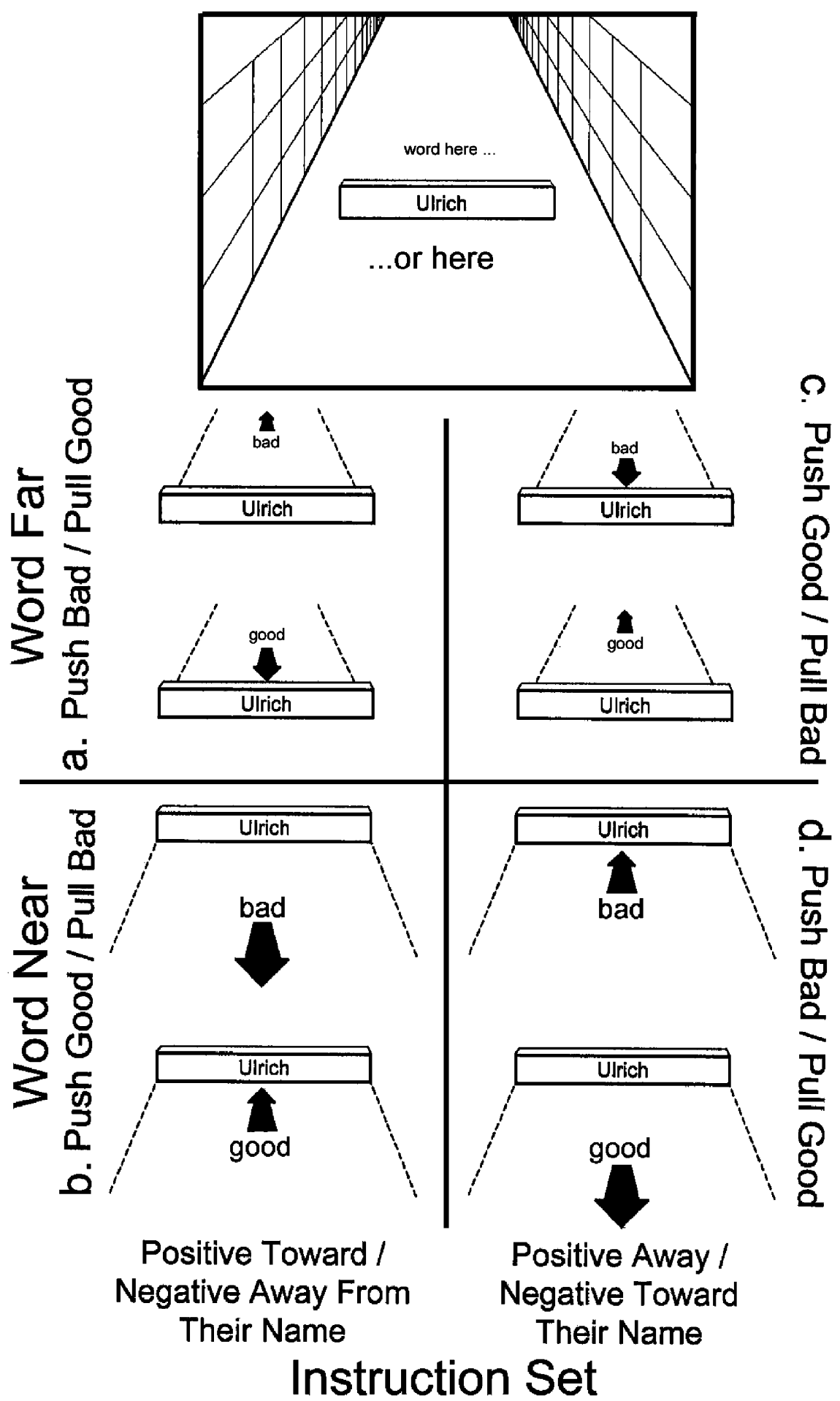

Fig. 1. The experimental setup. The illustration at the top shows the corridor displayed on the computer monitor. The participant's name was presented in the center of the corridor, and a word to be evaluated was placed either further away or nearer than the name. Some participants were instructed to move the joystick in the direction from the word toward their name if the word was positive and to move the joystick in the direction away from their name if the word was negative; other participants were given the opposite instructions. As illustrated, the combination of instruction set and word location determined whether the participant pushed the joystick for positive words and pulled it for negative words or pushed the joystick for negative words and pulled it for positive words. When the word was presented near in the corridor, the participant's representations of self and body were deconfounded. Participants did not see the arrows shown here to symbolize the instructions for moving the words. 
TABLE 1

Mean Response Times (in Milliseconds) as a Function of Instruction Set, Movement Direction, and Valence

\begin{tabular}{llrr}
\hline \hline & \multicolumn{2}{c}{ Valence } & \\
\cline { 2 - 3 } Movement and instruction set & Positive words & Negative words & \multicolumn{1}{c}{ Mean } \\
\hline Pulling motion & $769(23.70)$ & $678(19.11)$ & $724(15.91)$ \\
$\quad$ Positive toward/negative away & $994(36.43)$ & $1,033(31.63)$ & $1,014(24.08)$ \\
$\quad \begin{array}{l}\text { Positive away/negative toward } \\
\text { Pushing motion }\end{array}$ & $723(23.83)$ & $697(21.10)$ & $710(15.88)$ \\
$\quad$ Positive toward/negative away & $966(30.72)$ & $1,031(36.88)$ & $999(24.11)$ \\
$\quad$ Positive away/negative toward & & & \\
\hline \hline
\end{tabular}

Note. Standard errors are in parentheses. Pulling versus pushing motions refer to moving the arm toward versus away from the body, respectively. See Figure 1 for an explanation of the instruction sets.

\section{RESULTS}

Response times from correct trials were log-transformed. Values more than 2 standard deviations from the mean were eliminated. Means used for this exclusion were calculated separately for each instruction-set condition. The data were then submitted to a 2 (valence) $\times 2$ (movement direction) $\times 2$ (instruction set) repeated measures analysis of variance (ANOVA).

In this analysis, a main effect of instruction set would indicate that the speed of participants' movements was driven by their representation of their selves rather than their representation of their bodies. Specifically, we predicted that participants would be faster to move positive words toward their name than to move positive words away from their name, and that they would be faster to move negative words away from their name than to move negative words toward their name. Consistent with this prediction, participants were significantly faster to respond to words in the positive toward/negative away condition $(M=717 \mathrm{~ms})$ than in the positive away/negative toward condition $(M=1,006 \mathrm{~ms}), F(1$, $89)=61.60, p<.001, \eta_{p}{ }^{2}=.41$. This effect held regardless of word valence or direction of movement, all $p \mathrm{~s}<.001$ (see Table 1).

If the speed of participants' movements was driven by their representation of their bodies rather than their representation of their selves, they would have been faster to pull than to push positive words and faster to push than to pull negative words. This pattern would result in an interaction between movement direction and valence. Surprisingly, there was a significant interaction between movement direction and valence, $F(1,267)=$ $7.80, p<.05, \eta_{p}{ }^{2}=.05$, but it reflected an effect in the opposite direction from that obtained by Chen and Bargh (1999). As shown in Table 2, participants were slightly faster $(t<1)$ to pull negative words toward their bodies than to push negative words away from their bodies. In contrast, they were significantly faster to push positive words away from their bodies than to pull positive words toward their bodies, $t(89)=3.61, p<.001$. This pattern is consistent with the results of Wentura et al. (2000) and Münsterberg (1892).

Finally, there was a significant interaction between instruction set and valence, $F(1,267)=20.92, p<.001, \eta_{p}{ }^{2}=.19$.
As shown in Table 1, participants were faster to make responses that involved moving the joystick away from their name ( $M=834 \mathrm{~ms}$ ) than to make responses that involved moving the joystick toward their name $(M=889 \mathrm{~ms})$. The source of this effect is not clear, but it does not alter the interpretation of the primary results of this study (see Brendl et al., in press, for more discussion about effects of this type).

\section{DISCUSSION}

Accounts of embodied phenomena, such as those of Barsalou et al. (2003) and Glenberg (1997), focus primarily on the connection between perceptual and action systems. These accounts do not distinguish between people's representation of their (nonphysical) selves and their bodies.

The present results constrain theories of embodied cognition by suggesting that the ease of a particular movement depends crucially on representations of the task that go beyond simple learned motor actions. We deconfounded people's bodies and nonphysical representation of themselves by placing their names in a visual scene of a corridor receding in depth. Participants were faster to move positive words toward their name (i.e., toward their representation of themselves) than away from their name regardless of whether this response required a pushing movement (which would push the word away from their physical body) or a pulling movement (which would pull the word toward their physical body). Neumann and Strack (2000) reached a similar conclusion based on the observation that motor and perceptual information prime approach or avoidance states.

\section{TABLE 2}

Mean Response Times (in Milliseconds) as a Function of Valence and Movement Direction

\begin{tabular}{lcc}
\hline \hline & \multicolumn{2}{c}{ Movement direction } \\
\cline { 2 - 3 } Valence & Pulling & Pushing \\
\hline Positive words & $885(24.90)$ & $848(23.33)$ \\
Negative words & $861(26.43)$ & $870(27.75)$ \\
\hline \hline
\end{tabular}

Note. Standard errors are in parentheses. 
The importance of people's representation of the task is consistent with findings from previous research on the Simon effect (e.g., Simon, 1990). In this effect, people are faster to respond with the hand that is on the same side as a directional cue provided by the stimulus, even if that directional cue is not relevant to the judgment being made. For example, if people are asked to respond with the left hand to a high tone and with the right hand to a low tone, they are faster to respond to tones played on their left when they use their left hand rather than their right hand, and they are faster to respond to tones played on their right when they use their right hand rather than their left hand, even though the spatial location of the tone is not relevant to the pitch judgment.

The Simon task confounds the physical movement made by participants with the physical location of the stimulus. That is, responses made with the left hand are also made to the participant's left. Studies have deconfounded these variables by having participants respond with their arms crossed, so that their right hand is pressing a button on their left side. When the task is done this way, the resulting Simon effect is based on the spatial compatibility of the stimulus and the button, rather than the spatial compatibility of the stimulus and the side of the body, so that responses to stimuli on the left are faster for buttons on the left than for buttons on the right, and vice versa (see Brendl, 2001; Wascher, Schatz, Kuder, \& Verleger, 2001). Our results are similar, because they suggest that people's representation of self is distinct from their low-level representation of particular aspects of their body.

Movement-compatibility effects demonstrate that evaluations and judgments that have been explained in terms of higherorder processes arise from important coupling of perceptual and motor systems. The information processing view of cognition often ignored these effects and their implications. Embodied accounts correct this oversight by emphasizing the importance of perceptual and motor representations in cognition. The present results suggest that perceptual and motor representations alone may not be sufficient to account for cognitive processing, because phenomena that at face value seem prime examples of lower-order perceptual and motor processing may nonetheless involve higher-order symbolic processing. At a minimum, embodied accounts must specify how symbolic information about the self is tied to perceptual and motor representations.

Acknowledgments-This research was supported by German Science Foundation grants given to C. Miguel Brendl. We thank Ulrich Beck, Monika Heller, and Joachim Vosgerau for their help with this project.

\section{REFERENCES}

Bargh, J.A., Chaiken, S., Govender, R., \& Pratto, F. (1992). The generality of the automatic activation effect. Journal of Personality and Social Psychology, 62, 893-912.
Barsalou, L.W. (1999). Perceptual symbol systems. Behavioral and Brain Sciences, 22, 577-660.

Barsalou, L.W., Niedenthal, P.M., Barbey, A.K., \& Ruppert, J.A. (2003). Social embodiment. Psychology of Learning and Motivation, 43, 43-92.

Brendl, C.M. (2001). Goals and the compatibility principle in attitudes, judgment, and choice. In G.B. Moskowitz (Ed.), Cognitive social psychology (pp. 317-332). Mahwah, NJ: Erlbaum.

Brendl, C.M., Markman, A.B., \& Messner, C. (in press). Indirectly measuring evaluations of several attitude objects in relation to a neutral reference point. Journal of Experimental Social Psychology.

Cacioppo, J.T., Priester, J.R., \& Bernston, G.G. (1993). Rudimentary determination of attitudes: II. Arm flexion and extension have differential effects on attitudes. Journal of Personality and Social Psychology, 65, 5-17.

Chen, S., \& Bargh, J.A. (1999). Consequences of automatic evaluation: Immediate behavior predispositions to approach or avoid the stimulus. Personality and Social Psychology Bulletin, 25, 215-224.

Dijksterhuis, A., \& Bargh, J.A. (2001). The perception-behavior expressway: Automatic effects of social perception on social behavior. Advances in Experimental Social Psychology, 33, 1-40.

Fazio, R.H., Sanbonmatsu, D.M., Powell, M.C., \& Kardes, F.C. (1986). On the automatic activation of attitudes. Journal of Personality and Social Psychology, 50, 229-238.

Glenberg, A.M. (1997). What memory is for. Behavioral and Brain Sciences, 20, 1-55.

Münsterberg, H. (1892). Die psychologische Grundlage der Gefühle [The psychological basis of the emotions]. In International Congress of Psychology, 2nd session (pp. 132-135). London: Williams \& Norgate.

Neumann, R., \& Strack, F. (2000). Approach and avoidance: The influence of proprioceptive and exteroceptive cues on encoding of affective information. Journal of Personality and Social Psychology, 79, 39-48.

Prinz, J.J. (2002). Furnishing the mind. Cambridge, MA: MIT Press.

Simon, J.R. (1990). The effects of an irrelevant directional cue on human information processing. In R.W. Proctor \& T.G. Reeve (Eds.), Stimulus-response compatibility: An integrated perspective (pp. 31-86). Oxford, England: North-Holland.

Solarz, A.K. (1960). Latency of instrumental responses as a function of compatibility with the meaning of eliciting verbal signs. Journal of Experimental Psychology, 59, 239-245.

Strack, F., Martin, L.L., \& Stepper, S. (1988). Inhibiting and facilitating conditions of the human smile. Journal of Personality and Social Psychology, 54, 768-777.

Wascher, E., Schatz, U., Kuder, T., \& Verleger, R. (2001). Validity and boundary conditions of automatic response activation in the Simon task. Journal of Experimental Psychology: Human Perception and Performance, 27, 731-751.

Wentura, D., Rothermund, K., \& Bak, P. (2000). Automatic vigilance: The attention-grabbing power of approach and avoidance-related social information. Journal of Personality and Social Psychology, $78,1024-1037$.

Wilson, M. (2002). Six views of embodied cognition. Psychonomic Bulletin \& Review, 9, 625-636.

(RECEIVED 1/27/04; REVISION ACCEPTED 3/17/04) 